\title{
Effects of Ethanol on the Cerebellum: Advances and Prospects
}

\author{
Jia Luo ${ }^{1}$
}

Published online: 2 May 2015

(C) Springer Science+Business Media New York 2015

\begin{abstract}
Alcohol abuse causes cerebellar dysfunction and cerebellar ataxia is a common feature in alcoholics. Alcohol exposure during development also impacts the cerebellum. Children with fetal alcohol spectrum disorder (FASD) show many symptoms associated specifically with cerebellar deficits. However, the cellular and molecular mechanisms are unclear. This special issue discusses the most recent advances in the study of mechanisms underlying alcoholinduced cerebellar deficits. The alteration in GABAA receptor-dependent neurotransmission is a potential mechanism for ethanol-induced cerebellar dysfunction. Recent advances indicate ethanol-induced increases in GABA release are not only in Purkinje cells (PCs), but also in molecular layer interneurons and granule cells. Ethanol is shown to disrupt the molecular events at the mossy fiber - granule cell - Golgi cell (MGG) synaptic site and granule cell parallel fibers - PCs (GPP) synaptic site, which may be responsible for ethanol-induced cerebellar ataxia. Aging and ethanol may affect the smooth endoplasmic reticulum (SER) of PC dendrites and cause dendritic regression. Ethanol withdrawal causes mitochondrial damage and aberrant gene modifications in the cerebellum. The interaction between these events may result in neuronal degeneration, thereby contributing to motoric deficit. Ethanol activates doublestranded RNA (dsRNA)-activated protein kinase (PKR) and PKR activation is involved ethanolinduced neuroinflammation and neurotoxicity in the developing
\end{abstract}

Jia Luo

jialuo888@uky.edu

1 Department of Pharmacology and Nutritional Sciences, University of Kentucky College of Medicine, 132 Health Sciences Research Building, 1095 Veterans Drive, Lexington, KY 40536, USA cerebellum. Ethanol alters the development of cerebellar circuitry following the loss of PCs, which could result in modifications of the structure and function of other brain regions that receive cerebellar inputs. Lastly, choline, an essential nutrient is evaluated for its potential protection against ethanolinduced cerebellar damages. Choline is shown to ameliorate ethanol-induced cerebellar dysfunction when given before ethanol exposure.

The cerebellum is the motor coordination center of the central nervous system (CNS) and is also involved in cognitive processing and sensory discrimination. It has been well established that alcohol abuse causes cerebellar dysfunction. Permanent cerebellar deficits are often observed in alcoholics and the deficits persist even with abstinence from alcohol [1, 2]. Excessive alcohol exposure results in cerebellar ataxia and alterations in hand movements and speed when striking a target, impaired postural stability and balance, and slower attenuated foot tapping. In addition, the developing cerebellum is particularly vulnerable to the toxic effects of alcohol. Children with fetal alcohol spectrum disorder (FASD) show many symptoms associated specifically with cerebellar deficits $[3,4]$. Children and adolescents with a history of prenatal alcohol exposure display a reduction in cerebellar volume and a decrease in the size of the vermis [5]. This special issue discusses the most recent advances in the study of mechanisms underlying alcohol-induced cerebellar deficits.

The function of neurons in the cerebellar cortex is tightly controlled by GABAergic inhibitory inputs provided by specialized interneurons located in the granule and molecular layers. Alterations in $\mathrm{GABA}_{\mathrm{A}}$ receptor-dependent neurotransmission have been implicated in underlying ethanol-induced impairment of cerebellar function [6]. Valenzuela and Jotty 
review recent advances in the study of ethanol's effect on $\mathrm{GABA}_{\mathrm{A}}$ receptor-mediated neurotransmission in the cerebellar cortical circuits [7]. Initial studies focused on Purkinje cells (PCs), the sole output of the cerebellar cortex. These highly specialized GABAergic neurons provide powerful inhibitory input to deep cerebellar nuclei neurons, regulating their activity. Recent findings indicate that ethanol-induced increases in GABA release are not only in PCs but also in molecular layer interneurons and granule cells. Ethanol exposure increases GABA release at molecular layer interneuron-to-Purkinje cell synapses and also at reciprocal synapses between molecular layer interneurons. In granule cells, ethanol exposure both potentiates tonic currents mediated by extrasynaptic $\mathrm{GABA}_{\mathrm{A}}$ receptors and increases the frequency of spontaneous inhibitory postsynaptic currents mediated by synaptic $\mathrm{GABA}_{\mathrm{A}}$ receptors. Currently, there are two distinct models on how ethanol produces these effects. In one model, ethanol primarily acts by directly potentiating extrasynaptic $\mathrm{GABA}_{\mathrm{A}}$ receptors, including a population that excites granule cell axons and stimulates glutamate release onto Golgi cells. In the other model, ethanol acts indirectly by increasing spontaneous Golgi cell firing via inhibition of the $\mathrm{Na}^{+} / \mathrm{K}^{+}$ATPase, a quinidine-sensitive $\mathrm{K}^{+}$channel, and neuronal nitric oxide synthase.

Cellular and molecular mechanisms underlying ethanolinduced cerebellar ataxia are unclear. The mossy fibergranule cell-Golgi cell (MGG) and granule cell parallel fibers-Purkinje cells (GPP) synaptic sites are targets of ethanol, and alterations at these sites may result in cerebellar dysfunction and ataxia. Dar discusses the effect of ethanol on the potential molecular events at the MGG synaptic site and GPP synaptic site [8]. Ethanol induces neuronal nitric oxide synthase (NOS) inhibition at the MGG synaptic site which acts as a critical trigger for Golgi cell activation, leading to granule cell deafferentation. Concurrently, ethanol-induced inhibition of adenosine uptake at the GPP synaptic site produces adenosine accumulation which decreases glutamate release and leads to the profound activation of PCs. These molecular events at the MGG and GPP synaptic sites are mutually reinforcing and decrease the excitatory output of deep cerebellar nuclei. These may be the potential mechanisms underlying ethanol-induced cerebellar dysfunction and ataxia.

Both aging and alcohol abuse have deleterious effects on cerebellar-based motor functions such as balance, postural stability, and fine motion. The effects of aging may enhance the effects of alcohol on the cerebellum. Dlugos discuss the findings on ethanol-induced alterations to the dendritic arbor of the Purkinje cells in aging rats [9]. Ethanol causes dilation of the extensive smooth endoplasmic reticulum (SER) which precedes the dendritic regression. The component of the SER that was most affected by ethanol is the sarco/endoplasmic reticulum $\mathrm{Ca}^{2+}$ ATPase pump (SERCA) responsible for resequestration of calcium into the SER. Ethanol also causes decreases in SERCA pump levels and induces endoplasmic reticulum (ER) stress. Therefore, ethanol-induced ER stress within the SER of PC dendrites is a potential mechanism underlying dendritic regression.

Ethanol-induced cerebellar damages persist even after complete abstinence from drinking. In fact, ethanol withdrawal has shown to provoke a variety of neuronal and mitochondrial damage to the cerebellum. Jung reviews the mechanisms underlying ethanol withdrawal-induced cerebellar damages [10]. Upon ethanol withdrawal, excitatory neurotransmitter molecules such as glutamate are released in the cerebellum. Glutamate signals are projected to PCs through granular cells. This excitatory neuronal signal may promote an increase in intracellular $\mathrm{Ca}^{2+}$ levels and a decrease in a $\mathrm{Ca}^{2+}$-binding protein, resulting in the excessive entry of $\mathrm{Ca}^{2+}$ to the mitochondria. This causes a prolonged opening of the mitochondrial permeability transition pore and the overproduction of harmful free radicals, impeding adenosine triphosphate (ATP)-generating function. Ethanol withdrawal also causes aberrant gene modifications through altered DNA methylation, histone acetylation, or microRNA expression. The interaction between these events and molecules may result in neuronal degeneration, thereby contributing to motoric deficit observed in ethanol withdrawal.

Developmental ethanol exposure caused neurodegeneration which may underlie behavioral deficits observed in FASD. Ethanol activates double-stranded RNA (dsRNA)activated protein kinase (PKR) [11]. Li et al. investigate the role of PKR and its intracellular activator RAX in ethanol-induced neurodegeneration in the cerebellum [12]. By utilizing PKR-deficient (N-PKR-/-) mice, they study the RAX/PKR interaction and how this interaction is related to ethanol neurotoxicity in the developing cerebellum. Ethanol-induced brain/body mass reduction as well as cerebellar neuronal loss is significantly lower in N-PKR-/- mice than wild-type mice. Ethanol promotes interleukin-1 $\beta$ (IL-1 $\beta$ ) secretion, a master cytokine regulating inflammatory response. However, ethanol-promoted IL- $1 \beta$ secretion is abolished in N-PKR $-/-$ mice. Thus, PKR activation may be involved in ethanol-induced neuroinflammation and plays an important role in ethanol neurotoxicity in the developing cerebellum.

In addition to the loss of neurons, developmental ethanol exposure may cause alterations in the development of cerebellar circuitry. It has been well established that ethanol exposures during the early postnatal period induce death of PCs. A significant reduction of climbing fiber inputs to the surviving PCs has been characterized. There have been few studies, however, evaluating the electrophysiological characteristics of PCs subsequent to postnatal ethanol exposure. Light et al. investigate the effect of ethanol on the firing 
pattern of PCs in acute slice preparations on postnatal days 1315 [13]. PCs from rat pups treated with ethanol on postnatal days 4-6 show a significantly increased number of inhibitory postsynaptic potentials (IPSCs) and a larger hyperpolarizationactivated current (Ih). Ethanol induces a significant increase in the number of basket cells per PC as well as the volume of colocalized basket cell axonal membrane with PCs. In addition, ethanol significantly increases HCN1 channel volume colocalized to PC volume. Therefore, the cerebellar cortex that survives targeted postnatal ethanol exposure is dramatically altered subsequent to PC death. The alterations in the development of cerebellar circuitry following ethanol-induced loss of PCs could result in modifications of the structure and function of other brain regions that receive cerebellar inputs.

Since developmental exposure to ethanol causes severe damage to the cerebellum, it is important to identify potential neuroprotective agents to ameliorate ethanol toxicity. Bearer et al. investigate the protective effect of choline on the developing cerebellum [14]. Choline is an essential nutrient but many diets in the USA are choline deficient. They sought to determine whether choline supplementation prior to alcohol exposure can alleviate ethanolinduced impairment of cerebellar function. In their study, pregnant mice were deprived of choline from embryonic day 4.5. From postnatal days $1-5$, pups were treated with either choline or saline. From postnatal days 6-20, pups were exposed to ethanol $(6 \mathrm{~g} / \mathrm{kg})$. On postnatal day 30 , cerebellar function was tested using the dowel crossing test. Their study showed that choline ameliorated ethanolinduced cerebellar dysfunction when given before ethanol exposure. Therefore, choline supplement may reduce the toxic effects of ethanol in the developing cerebellum.

\footnotetext{
Acknowledgments This research is supported by a grant from National Institutes of Health (NIH) (AA015407). This work is also supported in part by the Department of Veterans Affairs, Veterans Health Administration, Office of Research and Development (BX001721) (Biomedical Laboratory Research and Development).
}

Conflicts of Interest The author has no conflicts to declare.

\section{References}

1. Sullivan EV, Desmond JE, Lim KO, Pfefferbaum A. Speed and efficiency but not accuracy or timing deficits of limb movements in alcoholic men and women. Alcohol Clin Exp Res. 2002;26:705-13.

2. Sullivan EV, Pfefferbaum A. Neurocircuitry in alcoholism: a substrate of disruption and repair. Psychopharmacology. 2005;180: 583-94.

3. Norman AL, Crocker N, Mattson SN, Riley EP. Neuroimaging and fetal alcohol spectrum disorders. Dev Disabil Res Rev. 2009;15: 209-17.

4. West JR. Acute and long-term changes in the cerebellum following developmental exposure to ethanol. Alcohol Alcohol Suppl. 1993;2:199-202.

5. Autti-Rämö I, Autti T, Korkman M, Kettunen S, Salonen O, Valanne L. MRI findings in children with school problems who had been exposed prenatally to alcohol. Dev Med Child Neurol. 2002;44:98-106.

6. Jaatinen P, Rintala J. Mechanisms of ethanol-induced degeneration in the developing, mature, and aging cerebellum. Cerebellum. 2008;7(3):332-47.

7. Valenzuela CF, Jotty K. Mini-review: effects of ethanol on GABAA receptor-mediated neurotransmission in the cerebellar cortex-recent advances. Cerebellum. 2015. doi:10.1007/s12311-014-0639-3.

8. Dar MS. Ethanol-induced cerebellar ataxia: cellular and molecular mechanisms. Cerebellum. 2015. doi:10.1007/s12311014-0638-4.

9. Dlugos CA. Ethanol-induced alterations in purkinje neuron dendrites in adult and aging rats: a review. Cerebellum. 2015. doi:10. 1007/s12311-014-0636-6.

10. Jung ME. Alcohol withdrawal and cerebellar mitochondria. Cerebellum. 2014. doi:10.1007/s12311-014-0598-8.

11. Chen G, Ma C, Bower KA, Ke Z, Luo J. Interaction between RAX and PKR modulates the effect of ethanol on protein synthesis and survival of neurons. J Biol Chem. 2006;281(23):1590915.

12. Li H, Chen J, Qi Y, Dai L, Zhang M, Frank JA, et al. Deficient PKR in RAX/PKR association ameliorates ethanol-induced neurotoxicity in the developing cerebellum. Cerebellum. 2015. doi:10.1007/ s12311-015-0644-1.

13. Light KE, Hayar AM, Pierce DR. Electrophysiological and immunohistochemical evidence for an increase in GABAergic inputs and HCN channels in Purkinje cells that survive developmental ethanol exposure. Cerebellum. 2015. doi:10.1007/s12311-0150651-2.

14. Bearer CF, Wellmann KA, Tang N, He M, Mooney SM. Choline ameliorates deficits in balance caused by acute developmental ethanol exposure. Cerebellum. 2015. (in this issue). 